\title{
Efficacy of Non-thermal Ultrasound in the Management of Breast Engorgement in Post-partum Women: A randomized controlled trial
}

\author{
Habibu W., Hanif S. \\ Department of Physiotherapy, Bayero University, Kano, Nigeria
}

\section{Correspondence}

Dr. Shmaila Hanif, Department of Physiotherapy, Faculty of Allied Health Sciences, College of Health Sciences, Bayero University Kano•e-mail: shelllshell@@yahoo.com; smhanif.pth@buk.edu.ng

\begin{abstract}
SUMMARY
Breast engorgement is one of the main reasons why women throughout the world stop or reduce the duration of breast feeding. The objective of the study, was to determine the efficacy of non-thermal ultrasound in the management of breast engorgement. Fifty-two postpartum women with breast engorgement were included in the study and were randomly assigned to an experimental group and a control group of 26 participants each. Pain and breast engorgement were assessed using the visual analogue scale (VAS) and the six-point engorgement scale (SPES), pre- and post-intervention in both groups. The experimental group received non-thermal therapeutic ultrasound therapy along with conventional treatment interventions for two days while the control group only received conventional treatment for two days. The control group received massage, advice on proper latching technique and the use of bra. Results indicate a significant $(\mathrm{P}<0.05)$ difference in symptoms of breast engorgement between pre-intervention and post-intervention in both groups, with greater reduction in symptoms in the experimental group. Also, a maximum of two days was found to be effective in the management of breast engorgement in the experimental group. The use of therapeutic non-thermal ultrasound for 3 treatment sessions in two successive days was effective in the management of breast engorgement and is recommended for use in physiotherapy clinical settings.
\end{abstract}

KEY WORDS: breast engorgement, non-thermal ultrasound, postpartum, pain

\section{INTRODUCTION}

Breast engorgement is a painful and unpleasant condition that affects a large number of women in the early postpartum period (Mathew, 2013). Mangesi and Dowswell (2010) explained that, it is the over-filling of breast milk that causes discomfort and pain to the mother. However, the distress associated with breast engorgement may mean that, women initiating breastfeeding may not persist beyond the first few days after birth (Mass, 2004), thus inhibiting the development of successful breastfeeding. This can lead to many breast conditions, such as infection of the breast (Mangesi and Dowswell, 2010).

Engorgement causes swelling around the nipple which makes it even more difficult for the baby to latch on and feed successfully, and this may make the engorgement worse. The breasts become hard, and the skin appears shiny and in tension. Pain and tenderness are the major characteristics, followed by a low-grade fever, an aching sensation, generalized malaise, numbness, and a tingling sensation in the hands that occurs as a result of pressure exerted by swelling on the nerves (Mass, 2004). These characteristics may be compounded if the baby is not suckling enough (Hillenbrand and Larsen, 2002).

A number of studies have been conducted, using different methods in the management of breast engorgement. These methods, which include cold cabbage 
compresses (Arora et al, 2008), cold gel pads (Spencer et al, 2012), hot compresses and warm showers (Armstrong et al, 2006), are used to activate the milk ejection reflex (Snowden et al, 2001). Further treatment methods which have been postulated include: the use of breast binding (Swift and Janke, 2003), breast massage (Snowden et al, 2001), anti-inflammatory medication (such as serrapeptase [Danzen] which reduces swelling) (Kee et al, 1989), kinesio-therapeutic taping technique (Brown and Langdon, 2014), and Gua-Sha therapy (Chiu et al, 2010) which involves scraping the skin with a massage tool. Some of these techniques either cause other complications or are invasive in nature.

The use of both thermal and non-thermal therapeutic ultrasound (McLachlan et al, 1991; Shellshear, 1981; Sankanagoudar et al, 2011) have long been advocated in the management of breast engorgement. Research conducted on non-thermal ultrasound reported its efficacy in the management of breast engorgement. However, some of these researches (McLachlan et al, 1991; Shellshear, 1981) have limitations of either a poor research design, poor documentation or poor sample size. Recommendations were made by McLachlan et al. (1991) to conduct more randomized trials with modification of dosage with respect to the pulsed mode.

Sankanagoudar et al. (2011), in a study on the management of breast engorgement with the use of nonthermal ultrasound reported, relief of symptoms following two days. Their study included administration of nonthermal ultrasound twice a day. The present study subjected the patients with breast engorgement to non-thermal ultrasound treatment three times a day. This is because, with the use of the conventional method treatment lasts more than just a day and breast engorgement, which is a painful condition, needs prompt and maximal approach.

This study therefore, aimed to authenticate the efficacy of non-thermal ultrasound in the management of breast engorgement.

\section{METHODOLOGY}

\section{Research Design}

The experimental design (randomized controlled trial) was adopted in this study as it represents the gold standard for evaluating the effectiveness of health care interventions and it allows for randomization (to prevent bias) to both the experimental and control groups.

\section{Population}

The population of the study, comprised all postpartum mothers diagnosed with breast engorgement in the postnatal ward of Aminu Kano Teaching Hospital (AKTH), Kano, a major referral centre located in the most populous Nigerian state, inhabited by over 9 million people (National Population Commission National Census, 2009).

\section{Sample Size and Sampling Technique}

A total of fifty-two (52) (using the Cohen table) post-partum women with breast engorgement, who delivered at AKTH, Kano, were selected out of post-partum women having breast engorgement. Random sampling was employed and subjects were randomly allocated to the experimental and control groups, giving twenty six in both. Allocation concealment, a procedure for the randomization process so that the treatment to which each patient is allocated is not known before the patient is entered into the study, was performed. The ballot method was used for this purpose. Participant selection was made based on the following inclusion and exclusion criteria:

Inclusion criterion: Postnatal mothers diagnosed with symptomatic post-partum breast engorgement.

Exclusion criteria: Mothers receiving lactation suppressants; postnatal mothers with breast infection (mastitis): this was ruled out at the initial stage of assessment at the Obstetrics and Gynaecology department; cancer of the breast: this will be ruled out from the history of each participant.

\section{Data Collection Instruments}

The following instruments were used for the study:

1. Ultrasound machine (ENRAF NONIUS/ Netherlands): This modality was used in the management of breast engorgement. It is an equipment for therapy that uses high frequency sound waves which can cause mechanical and thermal effects in the tissue. The neatly arranged touch-screen operating panel makes operation extremely easy and fast. All information needed is displayed in a well-organized manner.

2. Visual analogue scale (VAS): This was used to assess level of pain. The reliability and validity has been recorded to be $0.60-0.77$ and $0.76-0.84$ respectively (Taddio et al, 2009).

3. Six-point engorgement scale: This was used to assess the severity of breast engorgement. The reliability has been recorded to be 0.84 (Hill and Humenick, 1994). 
The responses of the participants were scored as follows: [1] for soft and no changes in breast, [2] for slight changes in the breast, [3] for firm and no tender breast, [4] for firm, and beginning tenderness in breast, [5] for firm and tender breast, and [6] for very firm and very tender. Scoring: The minimum score in this scale is 1 and the maximum is 6 . The severity of breast engorgement is categorized as mild (scores 1-3), moderate (scores 4-5) or severe (score of 6).

4. Stadiometer (SECA gmbh/Germany): This will be used to measure the stature of participants.

5. Portable weighing scale (SECA gmbh/Germany): This was used to determine the body mass (BM) of participants.

6. Tape measure (Butterfly/China): This was used to determine the cup size of a participant's breast.

\section{Data collection procedure}

Before commencement of the study, ethical approval was sought from the Ethical Committee of AKTH, Kano. Participants who were diagnosed as having breast engorgement by the Obstetrics \& Gynecology department were selected. All participants or their proxies were asked to sign the informed consent form after details of the study had been explained to them. Data was collected in the Physiotherapy Department, AKTH, Kano.

Based on the inclusion criterion, 52 subjects were selected and randomized into the experimental group and the control group, each comprising 26 participants.

Group A: Experimental group (Non-thermal ultrasound) Group B: Control group (Conventional treatment)

The following measurements were taken and recorded:

1. Stature: The stadiometer was mounted on a straight wall that is at a true $90^{\circ}$ angle to the floor. The heel plate was mounted on the floor in the same vertical plane as the backboard of the stadiometer. Each participant stood with heels together, barefoot or wearing thin socks or stockings and relaxed, with their back against the wall-mounted stadiometer. The horizontal bar was adjusted down to rest firmly on the top of the head and a weight of about $0.5 \mathrm{~kg}$ was placed on the headboard. The measurement was recorded on the counter (Stewart and Eston, 2006).

2. Body mass: The participant weights were measured using a standard weighing scale with provision for calibration. Subjects were asked to present themselves in light clothing and to remove all heavy objects prior to measurement. They were then instructed to step on the weighing scale barefooted and stand erect, with the face looking straight forward and their hands by the side. The reading was then taken and recorded to the nearest $0.5 \mathrm{~kg}$ (Florey, 1970).

3. Body mass index (BMI): BMI is computed by dividing the participant's weight in kilogrammes with the square of their height in meters (Janssen et al, 2005).

$$
\mathrm{BMI}=\text { Body mass }(\mathrm{kg}) /[\text { Stature }(\mathrm{m})]^{2}
$$

4. Breast cup size: This was determined using a tape measure. Breast cup size was assessed by measuring the horizontal chest circumference just under the breasts, adding 5 inches $(12.7 \mathrm{~cm})$ and subtracting the total from the horizontal chest circumference at the fullest part of the breasts. A net difference of 1 inch is equivalent to A cup size, 2 inches is $\mathrm{B}$ cup size, 3 inches is $\mathrm{C}$ cup size and 4 inches is D cup size (Noble and Noble, 1998).

5. Pain: Pain associated with breast engorgement was assessed using VAS. A constructed line of $10 \mathrm{~cm}$ drawn on a sheet of paper was presented to the patients. At one end of the paper was written 'no pain' and at the other end 'severe pain'. The patients were instructed to mark the point corresponding to the intensity of their pain at that very moment. The reading was obtained by rating the line with a ruler, to determine the exact point that corresponds with the patient's pain perception in $\mathrm{cm}$ (Jensen et al, 1998).

Scoring: $0-0.4 \mathrm{~cm}=$ No pain; $0.5-4.4 \mathrm{~cm}=$ Mild pain; $4.5-7.4 \mathrm{~cm}=$ Moderate pain; $7.5-10 \mathrm{~cm}=$ Severe pain.

6. Breast engorgement: This was assessed using the sixpoint engorgement scale. It is a standardized tool to assess the occurrence of breast engorgement during the first, second and third day of the postnatal period. The reliability of the tool has been recorded as 0.84 (Hill and Humenick, 1994).

Scoring: Minimum score in this scale is 1 and the maximum is 6. Severity of breast engorgement is categorized as follows:

$$
\begin{aligned}
& \text { Mild }-1-3(\text { coded as } 1) \\
& \text { Moderate }-4-5(\text { coded as } 2) \\
& \text { Severe }-6(\text { coded as } 3)
\end{aligned}
$$

\section{Treatment Protocol}

The pre- and post-treatment scores were recorded for each treatment session. Participants were randomly assigned to two groups: experimental group and control group. 


\section{Experimental group (Group A)}

This group received both non-thermal ultrasound and conventional treatment three times daily. The treatment protocol for non-thermal ultrasound was as follows:

i. Patient position: The participant assumed supine position with a pillow under the head and hands behind the head.

ii. Patient preparation: The breast was cleaned using cotton wool and $70 \%$ alcohol solution for sterilization.

iii. Procedure: The breast was demarcated into four quadrants, and each quadrant was treated for a specific time. Treatment with non-thermal ultrasound was given using an aquasonic gel as the coupling medium on the breast and the transducer head. The transducer head was massaged over the breast towards the areola using firm pressure on inward strokes (McLachlan et al, 1991).

iv. Treatment parameters: Mode - Pulsed; Frequency $-1 \mathrm{MHz}$; Intensity $-0.5 \mathrm{~W} / \mathrm{cm}^{2}$

v. Duration of treatment: This depended on the bra cup size (Sankangoudar et al, 2011):

- A cup size - 10mins (2.5mins for each quadrant)

- B cup size - 12 mins (3mins for each quadrant)

- C cup size - 14mins (3.5mins for each quadrant)

- D cup size - 15mins (3.75mins for each quadrant)

vi. Termination of treatment: The breast was cleaned using $70 \%$ alcohol solution and cotton wool to remove the aquasonic gel used. The participant was asked to report any feelings. She was then reminded to wash the breast before breastfeeding.

vii. Precaution: It was ensured that the applicator was moved constantly throughout the treatment to avoid the damaging effect of standing wave.

\section{Control group (Group B)}

This group received conventional treatment. The treatment comprised:

1. Massage: A conventional treatment for relieving the symptoms of breast engorgement which aids in expression of milk. Kneading and effleurage are the best techniques (Sankanagoudar et al, 2011).

a. Participant position: The participant assumed supine position with a rolled towel under the head and the hands by the side.

b. Procedure: The palms were made warm by rubbing them together. The breast was kneaded in circular motion using the fingers encircling the breast from the nipple outwards. Strokes were then used also from the nipple outwards (McLachlan et al, 1991).

c. Treatment parameters: Frequency: three times daily.

2. Proper latching technique: The subject was asked to hold the baby with the whole body facing her body. The baby's chin and nose were placed against the breast with the participant supporting the head, neck and back of the baby with the arm. The baby was drawn to the nipple and areolar placed into the mouth (McLachlan et al, 1991).

3. Proper fitting bra: The participant was advised on the use of bra. The fitting of the bra should neither be too tight nor too loose (Noble and Noble, 1998).

\section{Data Analysis Procedure}

Descriptive and inferential statistics were used to analyse the data. Descriptive statistics of simple frequency, percentage, mean and standard deviation were computed for subjects' stature, body mass, and body mass index. The dependent t-test was computed to determine difference in pain scores before and after intervention in both the experimental and control groups. Mann-Whitney U was computed to determine the difference in breast engorgement scores before and after intervention in both the experimental and control groups. The independent t-test was used to determine differences in the baseline and post-intervention pain scores in both the experimental and control groups. The Wilcoxon signed rank test was used to determine the difference in baseline and post-intervention breast engorgement between the experimental and control groups. All the statistical analyses were performed using the Statistical Package for Social Sciences- SPSS (Windows version 16.0, Chicago, IL, USA). A probability level of 0.05 was used to indicate statistical significance.

\section{RESULTS}

A total of 52 postpartum women participated in this study. Twenty-six (26) of the women were subjected to nonthermal ultrasound therapy while the other 26 were subjected to conventional therapy in the form of massage, advice on proper lactation technique and proper fitting of bra.

The physical characteristics of the participants is displayed in table 1 . On the average, participants in both groups fell within the normal BMI category. 
Table 1. Physical characteristics of participants

\begin{tabular}{lcccc}
\hline Variables & $\begin{array}{c}\text { Control Gp } \\
\mathrm{M} \pm \mathrm{SD}\end{array}$ & $\begin{array}{c}\text { Experimental Gp } \\
\mathrm{M} \pm \mathrm{SD}\end{array}$ & t-value & $\begin{array}{c}\mathrm{P} \text { - } \\
\text { value }\end{array}$ \\
\hline Stature $(\mathrm{m})$ & 1.588 & 1.622 & -1.995 & 0.029 \\
$\mathrm{BM}(\mathrm{Kg})$ & 51.580 & 55.580 & -1.743 & 0.755 \\
$\mathrm{BMI}\left(\mathrm{Kg} / \mathrm{m}^{2}\right)$ & 20.409 & 21.156 & -0.960 & 0.933 \\
\hline
\end{tabular}

The pre-intervention pain level and post-intervention pain level of the experimental group and the control group are presented in table 2 . The results show that there is no significant $(\mathrm{P}>0.05)$ difference in pre-intervention pain between the control group and the experimental group. However, there is a significant $(\mathrm{P}<0.05)$ difference in post-intervention pain between the control group and the experimental group. The mean post-intervention value is higher in the control group compared to the experimental group. This implies that following intervention, there was a reduction in pain in both the control and experimental groups, with the experimental group having almost complete recovery from pain.

Table 2. Summary of the independent t-test showing difference in baseline and post intervention pain scores in both the experimental and control groups

\begin{tabular}{lccrl}
\hline Variables & $\begin{array}{c}\text { Control Gp } \\
\mathrm{M} \pm \mathrm{SD}\end{array}$ & $\begin{array}{c}\text { Experimental Gp } \\
\mathrm{M} \pm \mathrm{SD}\end{array}$ & t-value & $\begin{array}{l}\mathrm{P}- \\
\text { value }\end{array}$ \\
\hline Pre-intervention & $5.19 \pm 0.981$ & $5.69 \pm 1.569$ & -1.378 & 0.174 \\
Post-intervention & $2.69 \pm 1.011$ & $0.35 \pm 0.629$ & $10.050^{*}$ & 0.009 \\
\hline $\mathrm{t}_{(50)}=2.009 \cdot \mathrm{P}<0.05$ & & &
\end{tabular}

The breast engorgement levels before and after intervention are displayed in table 3. There are no significant $(\mathrm{P}>0.05)$ differences in the pre-intervention and post-intervention breast engorgement values between the control group and the experimental group.

Table 3. Summary of Mann-Whitney U showing difference in breast engorgement (BE) scores pre-intervention and post-intervention

\begin{tabular}{lllllll}
\hline Variables & Groups & $\begin{array}{l}\text { Mean } \\
\text { rank }\end{array}$ & $\begin{array}{l}\text { Sum of } \\
\text { ranks }\end{array}$ & P-value \\
\hline Pre-intervention BE & Experimental & 20.00 & 754.00 & 273.000 & 0.158 \\
& Control & 24.00 & 624.00 & & \\
Post-intervention BE & Experimental & 25.50 & 663.00 & 312.000 & 0.153 \\
& Control & 27.50 & 715.00 & & \\
\hline
\end{tabular}

The differences in the pain scores of both groups are displayed in table 4 . The results show significant $(\mathrm{P}<0.05)$ differences between pre-intervention pain and post intervention pain in both the control and experimental groups. The mean pain values decreased following intervention in both groups, implying that there was improvement in pain. However, the mean post-intervention pain value is lower and closer to the "no pain" category in the experimental group.

Table 4. Summary of Dependent t-test showing difference in pain scores of both groups

\begin{tabular}{llcrr}
\hline Group & Variable & $\mathrm{M} \pm \mathrm{SD}$ & t-value & P-value \\
\hline Experimental & $\begin{array}{l}\text { Pre intervention } \\
\text { pain }\end{array}$ & $5.69 \pm 1.569$ & $18.233^{*}$ & 0.000 \\
& $\begin{array}{l}\text { Post-intervention } \\
\text { pain }\end{array}$ & $0.35 \pm 0.629$ & & \\
& $\begin{array}{l}\text { Pre-intervention } \\
\text { pain }\end{array}$ & $5.19 \pm 0.891$ & $18.028^{*}$ & 0.000 \\
& $\begin{array}{l}\text { Post-intervention } \\
\text { pain }\end{array}$ & $2.69 \pm 1.011$ & & \\
& & & & \\
\hline
\end{tabular}

$\mathrm{t}_{(25)}=1.708 ; \mathrm{P}<0.05$

The summary of Wilcoxon signed ranked test is displayed in table 5. The figures show significant $(\mathrm{P}<0.05)$ differences between the pre-intervention and post-intervention breast engorgement scores in both the experimental and control groups.

Table 5. Summary of Wilcoxon signed rank test showing the differences in baseline and post-intervention breast engorgement between the groups

\begin{tabular}{llllcl}
\hline Groups & Variables & $\begin{array}{l}\text { Mean } \\
\text { rank }\end{array}$ & $\begin{array}{l}\text { Sum of } \\
\text { ranks }\end{array}$ & $\mathrm{Z}$ & $\begin{array}{l}\text { P- } \\
\text { value }\end{array}$ \\
\hline $\begin{array}{l}\text { Experimental } \\
\text { group }\end{array}$ & $\begin{array}{l}\text { Pre intervention } \\
\text { BE }\end{array}$ & 12.00 & 276.00 & $-4.460^{*}$ & 0.000 \\
& $\begin{array}{l}\text { Post- } \\
\text { intervention BE }\end{array}$ & 0.00 & 0.00 & & \\
Control group & $\begin{array}{l}\text { Pre- } \\
\text { intervention BE }\end{array}$ & 9.50 & 171.00 & $-4.066^{*}$ & 0.000 \\
& $\begin{array}{l}\text { Post- } \\
\text { intervention BE }\end{array}$ & 0.00 & 0.00 & & \\
\hline & & & & & \\
\hline
\end{tabular}

\section{DISCUSSION}

This study was conducted to determine the efficacy of nonthermal ultrasound in the management of breast engorgement in postpartum women. There are limited solutions available to relieve the discomfort associated with breast engorgement instantly without any added complications of pain as in use of the Gua-sha therapy (Chiu et al, 2010) and breast pump (Snowden et al, 2001). Pain is a major problem that interferes with successful breastfeeding, leading to exclusive abandonment of 
breastfeeding that results in premature weaning at the early stage of lactation, that is three to five days after delivery (Mangesi and Dowswell, 2010).

All selected subjects participated in the study but some of the participants did not complete the treatment sessions (3 treatment sessions a day for 2 days). The reason for termination of treatment before completion of the study was due to complete resolution of symptoms and satisfaction from treatment. No participant reported any uncomfortable feeling or adverse effect of treatment with non-thermal ultrasound. All the treatment sessions were safe.

The use of non-thermal ultrasound is effective in relieving breast engorgement. This finding is similar to that of Sankanagoudar et al. (2011). However, the current study reported that the use of non-thermal ultrasound for three treatment sessions in two successive days resolves engorgement completely. This is contrary to the findings of Sankanagoudar et al. (2011).

An important observation was made in the course of this study; milk flow was found to occur during the application of non-thermal ultrasound. This finding was not reported in previous studies (Shellshear, 1981; McLachlan et al, 1991; Snowden et al, 2001; Sankanagoudar et al, 2011) on the use of therapeutic ultrasound. This possibly occurred due to the difference in the parameters used in this study.

The present study considered timing/duration of application of non-thermal ultrasound with respect to cup size which is a limitation of several previous studies (Sankanagoudar et al, 2011; Snowden et al, 2001).

\section{CONCLUSION AND RECOMMENDATION}

The use of therapeutic non-thermal ultrasound is safe and effective in the management of postpartum breast engorgement. Therapeutic non-thermal ultrasound should be used three times a day for a period of at least two days for the management of breast engorgement. Obstetricians should be made aware of the role of physiotherapy in the management of breast engorgement, so that such cases can be referred for physiotherapy.

\section{References}

Armstrong M., Caliendo C. and Roberts A. 2006. Pregnancy, lactation and nipple piercings. AWHONNLifelines 10(3): 212217.

Arora S., Vatsa M. and Dadhwal V. 2008. A comparison of cabbage leaves vs. hot and cold compresses in the treatment of breast engorgement. Indian Journal of Community Medicine 33(3): 160-162.
Brown D. and Langdon C. 2014. Does kinesio elastic therapeutic taping decrease breast engorgement in postpartum women? Clinical Lactation 5(2): 67-74.

Chiu J.Y., Gau M.L., Kuo S.Y., Chang Y.H., Kuo S.C. and Tu H.C. 2010. Effects of Gua-Sha therapy on breast engorgement: A randomized controlled trial. The Journal of Nursing Research 18(1): 1-10.

Florey C. 1970. The use and interpretation of ponderal index and other weight-height ratios in epidemiological studies. Journal of Chronic Diseases 23(2): 93-103.

Hill P.D. and Humenick S.S. 1994. The occurrence of breast engorgement. Journal of Human Lactation 10(2): 79-86.

Hillenbrand K.M. and Larsen P.G. 2002. Effect of an educational intervention about breastfeeding on the knowledge, confidence, and behaviors of pediatric resident physicians. Pediatrics 110(5): 59.

Janssen I., Katzmarzyk P.T., Boyce W.F., Vereecken C., Mulvihill C., Roberts C., Currie C. and Pickett W. 2005. Comparison of overweight and obesity prevalence in school aged youth from 34 countries and their relationships with physical activity and dietary patterns. Obesity Reviews 6(2): 123-132.

Jensen M.P., Miller L. and Fisher L.D. 1998. Assessment of pain during medical procedures: a comparison of three scales. The Clinical Journal of Pain 14(4): 343-349.

Kee W.H., Tan S.L., Lee V. and Salmon Y.M. 1989. The treatment of breast engorgement with Serrapeptase (Danzen): a randomised double-blind controlled trial. Singapore Medical Journal 30(1): 48-54.

Mangesi L. and Dowswell T. 2010. Treatments for breast engorgement during lactation. Cochrane Database Systematic Reviews 9, CD006946.

Mass S. 2004. Breast pain: engorgement, nipple pain and mastitis. Clinical Obstetrics and Gynecology 47(3): 676-682.

Mathew L.A. 2013. Effectiveness of cold cabbage leaves vs hot application on breast engorgement among postnatal mothers in a selected hospital, Bangalore. Sahyadri College of Nursing.

McLachlan Z., Milne E., Lumley J. and Walker B.L. 1991. Ultrasound treatment for breast engorgement: A randomised double blind trial. Australian Journal of Physiotherapy 37(1): 23-28.

National Population Commission National Census. 2009. Provisional figures. Federal Republic of Nigeria, 2010.

Noble C. and Noble D. 1998. Self-supporting breast cup. United States Patent 5755611.

Sankanagoudar P.P., Patil C. and Sirigeri K. 2011. Effect of therapeutic non-thermal ultrasound on post-partum symptomatic breast engorgement. Indian Journal of Physiotherapy and Occupational Therapy 5(2): 108113.

Shellshear M. 1981. Therapeutic ultrasound in post-partum breast engorgement. Australian Journal of Physiotherapy 27(1): 15-16.

Snowden H., Renfrew M.J. and Woolridge M. 2001. Treatments for breast engorgement during lactation. 
Cochrane Pregnancy and Childbirth Group. 10.1002/14651858.CD000046.

Spencer R., Hinsliff-Smith K. and Walsh D. 2012. An exploration of infant feeding experiences of women in Lincolnshire in the early postnatal period. Project. The University of Nottingham.

Stewart A.D. and Eston R. 2006. Surface anthropometry. Sport and Exercise Physiology Testing Guidelines 76.

Swift K. and Janke J. 2003. Breast binding - Is it all that it's wrapped up to be? Journal of Obstetric, Gynecologic \& Neonatal Nursing 32(3): 332-339.

Taddio A., O’Brien L., Ipp M., Stephens D., Goldbach M. and Koren G. 2009. Reliability and validity of observer ratings of pain using the visual analog scale (VAS) in infants undergoing immunization injections. Pain 147(1): 141-146. 